\title{
Carrier Trap Parameters in NaI with Tl, In, and Eu Dopants
}

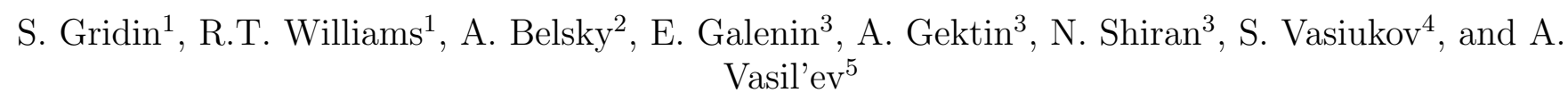

${ }^{1}$ Department of Physics, Wake Forest University, Winston-Salem, NC 27106, USA

${ }^{2}$ Institute of Light and Matter, CNRS, Claude Bernard University Lyon 1, 69100 Villeurbanne, France

${ }^{3}$ Institute for Scintillation Materials of the NAS of Ukraine, 61001 Kharkiv, Ukraine

${ }^{4}$ Istituto Nazionale di Fisica Nucleare, Sezione di Padova, 35131 Padova, Italy

${ }^{5}$ Skobeltsyn Institute of Nuclear Physics, Moscow State University, 119991 Moscow, Russia 
Table of model parameters used to simulate the TSL experiment in NaI:Tl, NaI:In, and NaI:Eu.

\begin{tabular}{|c|c|c|c|c|c|}
\hline Term meaning & Symbol & NaI:Tl value & NaI:In value & NaI:Eu value & Units \\
\hline Rate of e-h formation & $\alpha$ & $2 \times 10^{15}$ & $2 \times 10^{15}$ & $2 \times 10^{15}$ & $\mathrm{~cm}^{-3} \mathrm{sec}^{-1}$ \\
\hline Initial conc. of activator & $\mathrm{n}_{A}^{0}$ & $2 \times 10^{18} ; 5 \times 10^{18}$ & $7 \times 10^{17} ; 4.4 \times 10^{18}$ & $4.4 \times 10^{18} ; 2 \times 10^{19}$ & $\mathrm{~cm}^{-3}$ \\
\hline Initial conc. of STH sites & $\mathrm{n}_{S T H}^{0}$ & $1 \times 10^{20}$ & $1 \times 10^{20}$ & $1 \times 10^{20}$ & $\mathrm{~cm}^{-3}$ \\
\hline Initial conc. of defects ( $\mathrm{e}^{-}$traps) & $\mathrm{n}_{d}^{0}$ & - & $1 \times 10^{16}$ & - & $\mathrm{cm}^{-3}$ \\
\hline STH formation rate & $\beta_{S T H}$ & $3 \times 10^{-6}$ & $1 \times 10^{-13}$ & $3 \times 10^{-6}$ & $\mathrm{~cm}^{3} \mathrm{sec}^{-1}$ \\
\hline Rate of reaction $e+A^{+} \rightarrow A^{0}$ & $\beta_{e-A^{0}}$ & $2.5 \times 10^{-9}$ & 0 & $2.5 \times 10^{-9}$ & $\mathrm{~cm}^{3} \mathrm{sec}^{-1}$ \\
\hline Rate of reaction $h+A^{+} \rightarrow A^{2+}$ & $\beta_{e-A^{2+}}$ & $1 \times 10^{-9}$ & $1 \times 10^{-10}$ & $1 \times 10^{-10}$ & $\mathrm{~cm}^{3} \mathrm{sec}^{-1}$ \\
\hline Rate of reaction $e+d e f \rightarrow d e f^{e}$ & $\beta_{e-d}$ & - & $1 \times 10^{-9}$ & - & $\mathrm{cm}^{3} \mathrm{sec}^{-1}$ \\
\hline Rate of reaction $h+A^{0} \rightarrow A^{+*}$ & $\beta_{h-A^{0}}$ & $1 \times 10^{-8}$ & 0 & $1 \times 10^{-8}$ & $\mathrm{~cm}^{3} \mathrm{sec}^{-1}$ \\
\hline Rate of reaction $e+A^{2+} \rightarrow A^{+*}$ & $\beta_{e-A^{2+}}$ & $1 \times 10^{-5}$ & $1 \times 10^{-8}$ & $1 \times 10^{-5}$ & $\mathrm{~cm}^{3} \mathrm{sec}^{-1}$ \\
\hline Rate of reaction $e+S T H \rightarrow S T E$ & $\beta_{e-S T H}$ & $2 \times 10^{-6}$ & $1 \times 10^{-8}$ & $2 \times 10^{-6}$ & $\mathrm{~cm}^{3} \mathrm{sec}^{-1}$ \\
\hline Rate of reaction $h+d e f^{e} \rightarrow d e f^{*}$ & $\beta_{h-d}$ & - & $2 \times 10^{-5}$ & - & $\mathrm{cm}^{3} \mathrm{sec}^{-1}$ \\
\hline Freq. fact. of STH mobiliz. & $s_{S T H}$ & $3 \times 10^{13}$ & $3 \times 10^{10}$ & $3 \times 10^{13}$ & $\sec ^{-1}$ \\
\hline Activ. energy of STH mobiliz. & $E_{S T H}$ & 0.135 & 0.16 & 0.14 & $\mathrm{eV}$ \\
\hline Freq. fact. of $\mathrm{A}^{0}$ emptying & $s_{A^{0}}$ & $2 \times 10^{10}$ & 0 & $1 \times 10^{4}$ & $\sec ^{-1}$ \\
\hline Activ. energy of $\mathrm{A}^{0}$ emptying & $E_{A^{0}}$ & 0.26 & 1.0 & $0.05 ; 0.06$ & $\mathrm{eV}$ \\
\hline Freq. fact. of $\mathrm{A}^{2+}$ emptying & $s_{A^{2+}}$ & 0 & $2 \times 10^{10}$ & 0 & $\sec ^{-1}$ \\
\hline Activ. energy of $\mathrm{A}^{2+}$ emptying & $E_{A^{2+}}$ & 1.0 & 0.19 & 1.0 & $\mathrm{eV}$ \\
\hline Radiation time of $\mathrm{A}^{+*}$ & $\tau_{A^{+*}}$ & $0.6 \times 10^{-6}$ & $0.6 \times 10^{-6}$ & $0.6 \times 10^{-6}$ & sec \\
\hline Radiation time of STE & $\tau_{S T E}$ & $1.0 \times 10^{-6}$ & $1.0 \times 10^{-6}$ & $1.0 \times 10^{-6}$ & sec \\
\hline Radiation time of defects & $\tau_{d}$ & $1.0 \times 10^{-6}$ & $1.0 \times 10^{-6}$ & $1.0 \times 10^{-6}$ & sec \\
\hline Sample irradiation time at $10 \mathrm{~K}$ & - & 80 & 200 & 60 & sec \\
\hline Heating rate & - & 0.1 & 0.1 & 0.1 & $\mathrm{~K} \mathrm{sec}^{-1}$ \\
\hline
\end{tabular}

